\title{
Automated Determination of Optimal Angiographic Viewing Angles for Coronary Artery Bifurcations from CTA Data
}

\author{
Pieter H. Kitslaar ${ }^{\mathrm{a}}$, Henk A. Marquering ${ }^{\mathrm{a}}$, Wouter J. Jukema ${ }^{\mathrm{b}}$, Gerhard Koning ${ }^{\mathrm{a}}$, Maarten Nieber ${ }^{\mathrm{a}}$, \\ Albert M. Vossepoel ${ }^{\mathrm{c}}$, Jeroen J. Bax ${ }^{\mathrm{b}}$ and Johan H.C. Reiber ${ }^{\mathrm{a}}$ \\ ${ }^{a}$ Div. of Image Processing, Dept. of Radiology, Leiden University Medical Center, The Netherlands \\ ${ }^{b}$ Dept. of Cardiology, Leiden University Medical Center, Leiden, The Netherlands \\ 'Quantitative Imaging Group, Dept. of Imaging Science \& Technology, Faculty of Applied Sciences, \\ Delft University of Technology, The Netherlands
}

\begin{abstract}
For optimal diagnosis and treatment of lesions at coronary artery bifurcations using x-ray angiography, it is of utmost importance to determine proper angiographic viewing angles. Due to the increasing use of CTA as a first line diagnostic tool, 3D CTA data is more frequently available before x-ray angiographic procedures take place. Motivated by this, we propose to use available CTA data for the determination of patient specific optimal x-ray viewing angles.

A semi-automatic iterative region growing scheme is developed for the segmentation of the coronary arterial tree. From the segmented arterial tree, a complete hierarchical surface and centerline representation, including bifurcation points, is automatically obtained. The optimal viewing angle for a selected bifurcation is determined as the view rendering the least amount of foreshortening and vessel overlap.

For 83 bifurcation areas, viewing angles were automatically determined. The sensitivity of the method to patient positioning in the $\mathrm{x}$-ray system was also studied. Next, the automatically determined angels were both quantitatively and qualitatively compared with angles determined by two experts.

The method was found not to be sensitive to the positioning of the patient in the angiographic x-ray system. In $95 \%$ of the cases our method produced a clinically usable view (mean score of 8.4 out of 10 ) as compared to $98 \%$ for the experts (mean score of 8.7). Our method produced angiographic views with significantly less foreshortening (mean difference of 10 percentage points) than the angiographic views set by the experts.
\end{abstract}

Keywords: Cardiac Procedures, Treatment planning, Visualization

\section{INTRODUCTION}

Coronary x-ray angiographic images suffer from projection artifacts like foreshortening and vessel overlap. However, it is still the most suitable imaging modality that is usable for interventional procedures. Finding proper viewing angles for the x-ray system greatly improves the quality and decreases the duration of the interventional procedure.

A number of studies have been published on the reconstruction of the 3D coronary artery tree topology from multiple (2D) angiographic images to determine optimal viewing angles [1-4]. CTA data by definition contains this topological information obtained in a non-invasive way. Nowadays, CTA data is becoming more commonly available for patients undergoing coronary angiographic intervention. Motivated by this, we propose a method to obtain patient specific optimal angiographic viewing angles for coronary x-ray angiography based on available CTA data. From the CTA data the coronary trees can be segmented and used to simulate x-ray angiographic views. The amount of foreshortening and vessel overlap for a specific bifurcation region can be measured and used to determine the optimal viewing angle for that region.

The first part of this paper discusses the segmentation method to obtain the needed representation of the coronary tree for the optimal angle determination. Next, the method to obtain an optimal x-ray projection angle for a selected bifurcation in the coronary tree is discussed. Finally, some robustness and validation test are described and their results presented.

Medical Imaging 2008: Visualization, Image-guided Procedures, and Modeling, edited by

Michael I. Miga, Kevin Robert Cleary, Proc. of SPIE Vol. 6918, 69181J, (2008)

$1605-7422 / 08 / \$ 18 \cdot$ doi: $10.1117 / 12.770255$

Proc. of SPIE Vol. 6918 69181J-1 


\section{CORONARY TREE SEGMENTATION}

To determine the amount of foreshortening and vessel overlap, a centerline and surface representation of the coronary arterial tree is needed. Our segmentation method consists of a number of user guided iterative region growing steps. To speed up the region growing steps and prevent unwanted structures from being included in the final segmentation, first a pre-segmentation is performed. This pre-segmentation step removes large contrast filled (non coronary) regions in the $\mathrm{CT}$ datasets from being visited by the region grower.

\subsection{Pre-segmentation}

The large contrast filled structures in the CT dataset are removed using mathematical morphological operations[5]. A manually set threshold (commonly between 90 and $120 \mathrm{HU}$ ) is used to create a binary image of all the contrast filled regions in the $\mathrm{CT}$ dataset. This image is subsequently subsampled to decrease the computational time of the morphological operations. An erosion of the subsampled image is followed by a dilation removing the smaller structures (i.e. the coronary arteries) in the image. The size of structuring element for dilation was chosen slightly larger than that for the erosion to include an additional border around the large blood structures. The obtained binary mask is next resampled to the original image size to be used as a mask during the region growing step (see Fig. 1).
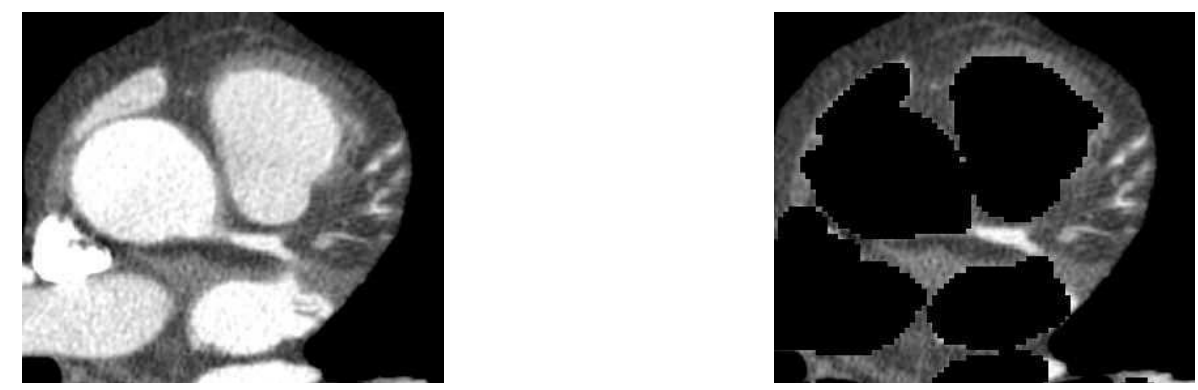

Fig. 1. Transversal slice of the CTA data before and after the masking of the large contrast filled regions.

\subsection{Region growing}

A region growing process is started using a user set seed point and threshold value to create an initial segmentation of the coronary tree. The seed point is placed in the ostium of the coronary tree. Since the large contrast filled regions are not visited by the region grower and the coronary structures are relatively small, the region growing step is performed very quickly. While the region grower is processing, it records the internal iteration number for each voxel it adds to the segmentation set. These iteration numbers can be seen as representations of the geodesic distance between the initial seed point and the voxels in the segmentation. Hierarchical information can next be extracted using these distance values (iteration numbers)[8]. This information can later be used to modify the segmentation result during additional region growing runs.

\subsection{Hierarchical analysis of the Coronary Tree}

The voxels in the segmentation are grouped in bins with a predefined distance interval (bin size), like in a histogram. An illustration of these histogram bins in a left coronary tree is shown in Fig. 2a. The histogram bins are labeled $H_{i}$ in ascending order based on their distance interval, with $0 \leq i \leq n_{b}$ and $n_{b}$ the total number of bins. Next, a connected components analysis is done on the voxels within each histogram bin. Each such connected component is called a segment and labeled $S_{i, j}$ with $i$ the index of the histogram bin containing the segment and $0 \leq j \leq n_{s}[i]$ with $n_{s}[i]$ the number of segments in $H_{i}$ (see Fig. 2b). Next a connectedness test is done between each segment in $H_{i}$ and each segment in $H_{i+1}$. If the segment $S_{i+1, k}$ is connected to segment $S_{i, j}$ then $P\left[S_{i+1, k}\right]=S_{i, j}$ is called the predecessor of segment $S_{i+1, k}$. For each segment $S_{i, j}$ its corresponding $P\left[S_{i, j}\right]$ is calculated. Using the predecessor list, $P$, the hierarchical graph representation of the segments in the coronary tree can be obtained.

The root node segment of the graph is the segment, $S_{0,0}$, containing the user set seed point. Next, the leaf point segments, $L_{i}$, in the tree can be determined. Since the region grower started in the root of the coronary tree, the voxel in the region grower output with the highest distance value (iteration number) is by definition a leaf point. From the segment, $S_{n, m}$, 
containing this point a trace back is made, using the predecessor list $P$, to the root segment $S_{0,0}$. All the traversed segments, $T\left[L_{0}\right]=\left\{S_{n, m}, S_{n-1, l}, \ldots, S_{0,0}\right\}$, in this trace back are "flagged" and stored for later use. The next leaf point is then found by searching for the voxel with the highest distance value but this time excluding voxels in the "flagged" segments. By repeating this procedure until all the segments have been flagged, a list of leaf points $L_{i}$ and corresponding trace backs $T\left[L_{i}\right]$ is obtained. Using the stored trace back list of each leaf point the vertex nodes (segments) in the graph can be determined. This is done by creating a reverse lookup list, $T^{*}\left[S_{i, j}\right]=\left\{L_{p}, \ldots, L_{q}\right\}$, from the $T\left[L_{0}\right], \ldots, T\left[L_{l}\right]$ lists, with $l$ the total number of leaf points (see Fig. 2c). From this list a set of unique combinations of leaf points can be extracted. Each set of leaf points in this list represents a node, $N_{i}=\left\{L_{p}, \ldots, L_{q}\right\}$, in the hierarchical tree graph.

If the leaf point set of $N_{i}$ contains multiple leaf points and this set is a subset of other node set, $N_{i}$ represents a vertex node. Furthermore, if the leaf point set of $N_{p}$, is the smallest possible set that contains the leaf point set of $N_{c}$, then node $N_{p}$ is the parent of node $N_{c}$ (see Fig. 2d). Naturally, the largest set represents the root point in the tree.

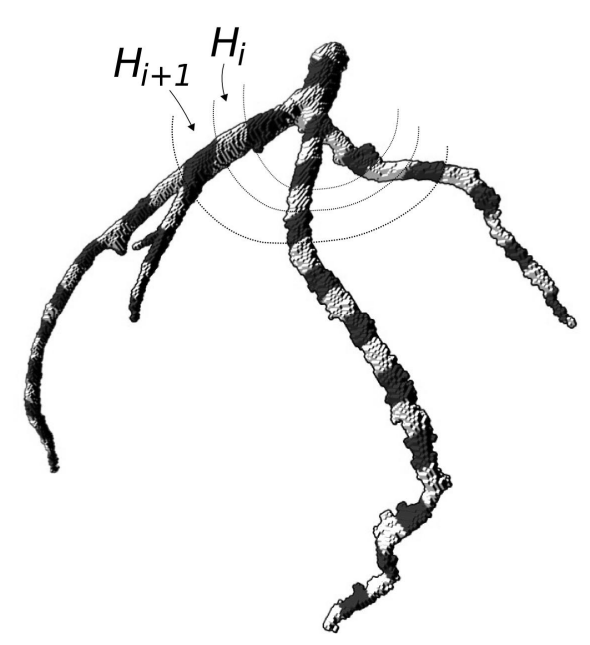

(a)

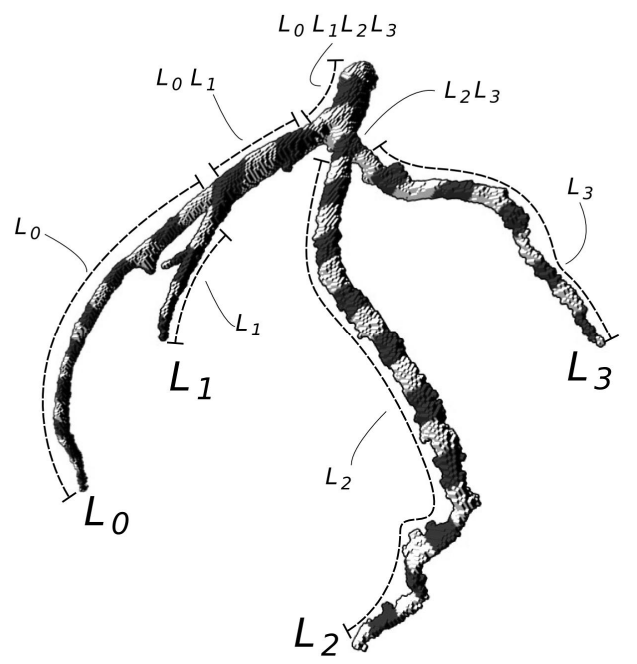

(c)

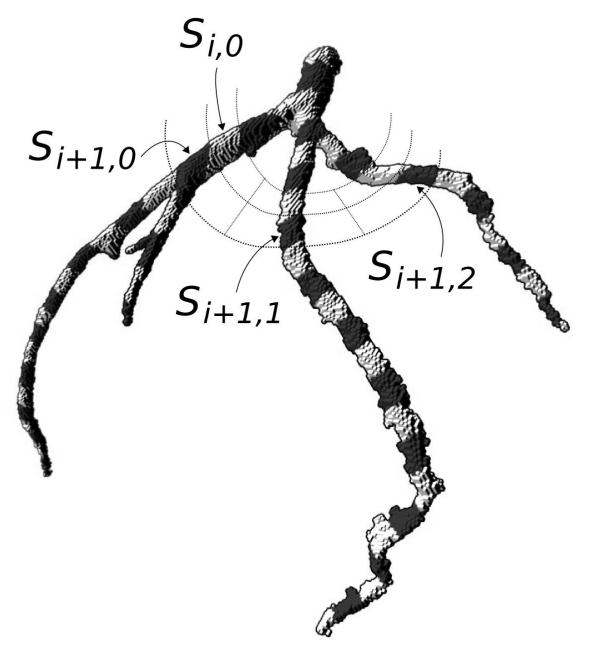

(b)

\begin{tabular}{|c|c|c|c|}
\hline Node & Leaf point set & Node Type & $\begin{array}{c}\text { Parent } \\
\text { node }\end{array}$ \\
\hline $\mathrm{N}_{0}$ & $\mathrm{~L}_{0} \mathrm{~L}_{1} \mathrm{~L}_{2} \mathrm{~L}_{3}$ & Root point & - \\
\hline $\mathrm{N}_{1}$ & $\mathrm{~L}_{0} \mathrm{~L}_{1}$ & Vertex point & $\mathrm{N}_{0}$ \\
\hline $\mathrm{N}_{2}$ & $\mathrm{~L}_{2} \mathrm{~L}_{3}$ & Vertex point & $\mathrm{N}_{0}$ \\
\hline $\mathrm{N}_{3}$ & $\mathrm{~L}_{0}$ & Leaf point & $\mathrm{N}_{1}$ \\
\hline $\mathrm{N}_{4}$ & $\mathrm{~L}_{1}$ & Leaf point & $\mathrm{N}_{1}$ \\
\hline $\mathrm{N}_{5}$ & $\mathrm{~L}_{3}$ & Leaf point & $\mathrm{N}_{2}$ \\
\hline $\mathrm{N}_{6}$ & $\mathrm{~L}_{4}$ & Leaf point & $\mathrm{N}_{2}$ \\
\hline
\end{tabular}

(d)

Fig. 2. Hierarchical analysis on region growing result. (a) 3D representation of the region grower segmentation showing histogram bins. (b) 3D representation of the region grower segmentation showing the segments derived from the histogram bins. (c) Hierarchy extraction from trace back segments. The $T^{*}\left[S_{i, j}\right]$ values are shown for the segments and the leaf points $L_{i}$. (d) Table showing the graph tree nodes based on the unique combinations of leaf points of figure $c$. The table also lists the node type and the parent node in the graph. 


\subsection{Multiple Region Growing Runs}

The above describe region growing and hierarchical analysis step may be repeated any number of times by the user to modify the segmentation. The user can modify the segmentation in two ways. Each run the threshold value used by the region grower may be modified to globally include more or less structures in the segmentation. Furthermore, the user is able to locally remove unwanted structures using the earlier calculated leaf points, $L_{i}$, and their corresponding set of trace back segments, $T\left[L_{i}\right]$. This is done by indicating which of the found leaf points are "accepted", $A_{L P}=\left\{L_{i}, \ldots, L_{k}\right\}$, and which are "rejected", $R_{L P}=\left\{L_{m}, \ldots, L_{n}\right\}$. The voxels in the segments of the set difference $R_{L P}-A_{L P}$ are removed from the segmentation. Furthermore, these voxels are added to a list of barred points. This list of barred points, like the presegmentation mask, prevents the region grower from including them during future region growing runs. The result of such a modification of the segmentation is shown in Fig. 3.

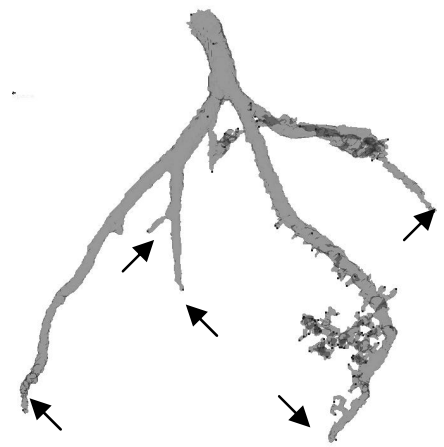

(a)

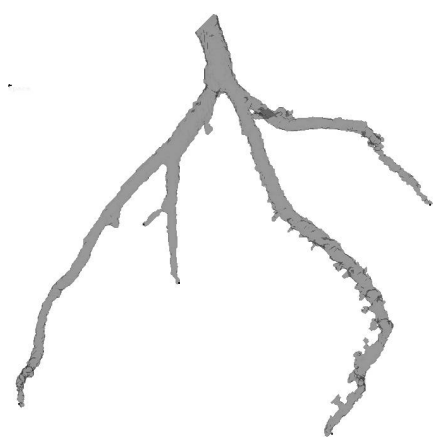

(b)

Fig. 3. Coronary tree segmentation. (a) Result of the region growing showing some unwanted leakages. The arrows indicate correctly grown leaf points as indicated by the user. (b) Region growing result one run later. Using the previously selected leaf points the leakages were removed from the segmentation.

\subsection{Surface and centerline representation}

When a satisfactory segmentation is obtained, a surface representation of the coronary tree is obtained from the segmentation using the marching cubes algorithm[9]. From the hierarchical tree analysis only the exact position of the root point and the leaf points is obtained. The location of the vertex points (bifurcations) in the coronary tree still have to be determined. First, the centerlines running from each leaf point of the coronary tree to the root point are calculated in the segmented voxel set using a fast wave propagation algorithm[7,8]. Next, for each bifurcation we use the two centerlines of its child nodes and the surface representation of the coronary tree to obtain a location for the corresponding bifurcation point. This bifurcation point is positioned at a certain distance from the carina of the bifurcation along a curve, called the average centerline, running between the two initial centerlines of the child nodes. We choose the position of the bifurcation point such that the distance to the carina equals half of the diameter of the common vessel segment at that distance $(d=0.5 \mathrm{~L}$ in Fig. 4). The diameter along the average centerline is calculated from the area of the cross section of the surface representation perpendicular to the average centerline. This bifurcation point is then used to calculate three new centerlines. Two centerlines run from the bifurcation point to its two child nodes and one centerline runs from the bifurcation point to the root point. This procedure is repeated for each bifurcation in the coronary tree, starting from the most distally located bifurcation upwards to the root.

When all the bifurcation points are obtained and all the centerlines are calculated (see Fig. 4c) the coronary tree representation is ready for the optimal projection angle determination. 


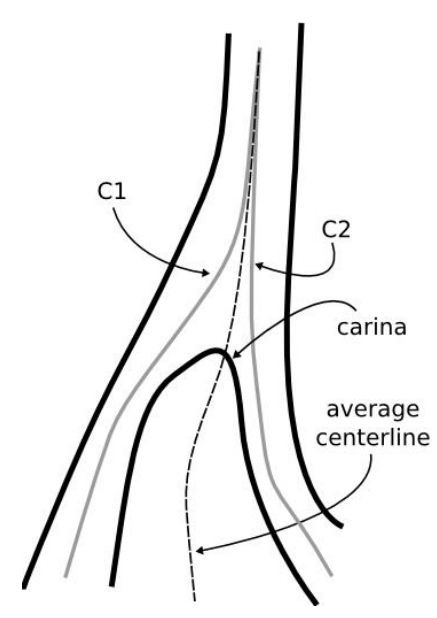

(a)

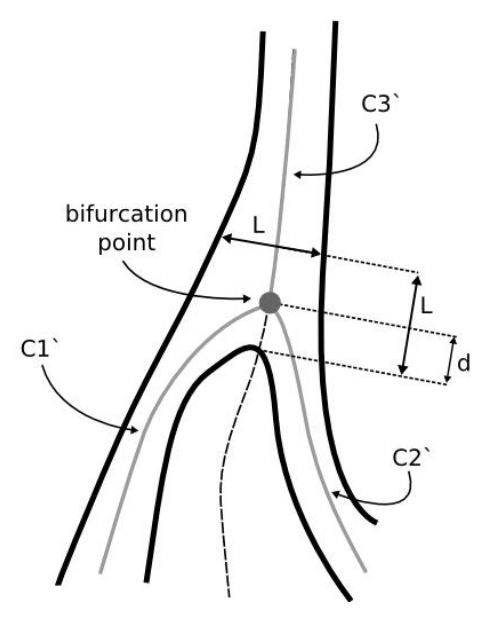

(b)

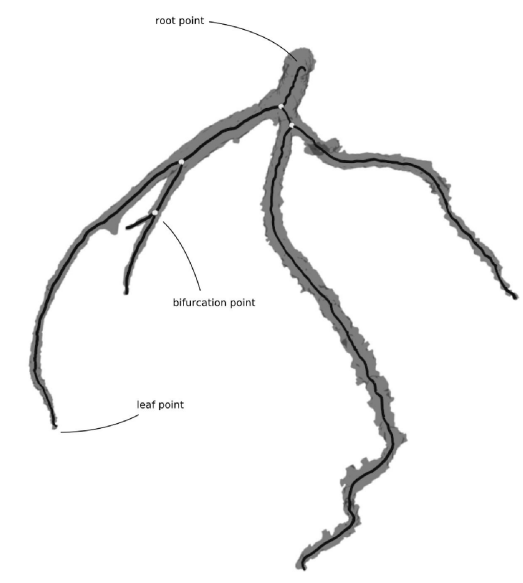

(c)

Fig. 4. (a) Schematic representation of bifurcation showing the initial centerlines $C 1$ and $C 2$, the average centerline and he carina of the bifurcation. (b) New centerlines $C 1$ ', $C 2$ ' and $C 3^{\prime}$ ' running through the bifurcation point positioned at a distance $d$ from the carina along the average centerline. $L$ indicates the diameter of the main vessel. (c) The final representation of the coronary tree showing the surface, centerlines and the bifurcation points.

\section{OPTIMAL ANGLE DETERMINATION}

In short, the optimal angle determination step consists of simulating x-ray projections of the selected bifurcation region for all possible projection angles of the x-ray angiographic system (see Fig. 5). Based on each simulated projection, a number of criteria are determined and stored for later analysis. The first criterion that is determined is the amount of foreshortening, based on the centerlines within the certain region of interest around the bifurcation point. Secondly, the amount of vessel overlap between a projection of the surface of the selected bifurcation region and the surface of the other parts of the coronary tree is determined. Up to this point our optimal angle determination method follows the approach of Chen et al.[3]. However, we introduce a third criterion, which we define as the "internal" overlap. For this, the three vessel branches of the bifurcation are separated from each other and the amount of overlap between these segments is calculated. This last criterion accounts for the fact that the bifurcation may overlap with itself. For the x-ray simulation a 7" diameter detector was assumed and the distance between the x-ray source and the isocenter and the detector and the isocenter is set to $75 \mathrm{~cm}$ and $40 \mathrm{~cm}$, respectively. Furthermore, the segmented coronary artery tree (patient in the x-ray system) was positioned in such a way that the bifurcation point under consideration lies in the angiographic isocenter of the simulated $\mathrm{x}$-ray system.

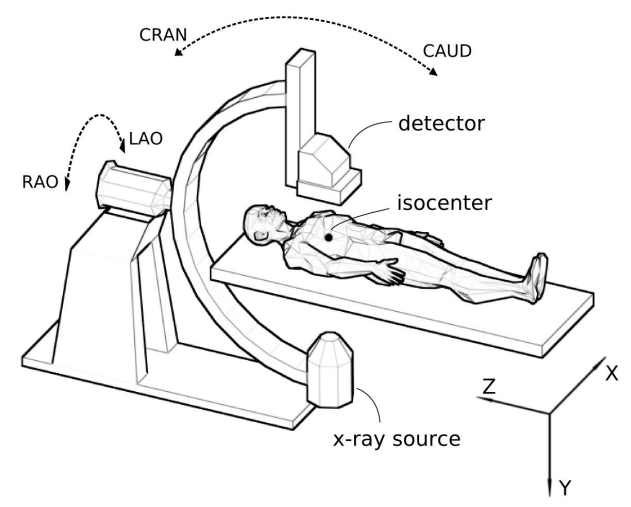

Fig. 5. Schematic representation of an x-ray angiographic system. 


\subsection{Region of interest and bifurcation splitting}

To determine the optimal view for a bifurcation in the coronary tree, a region of interest is defined as the first $10 \mathrm{~mm}\left(D_{r}\right.$ $=10 \mathrm{~mm}$ ) of the course of the arteries along the (three) centerlines from the bifurcation point (see Fig. 6a). This region of interest is again split at a distance, $D_{s}$, from the bifurcation point for later use in the internal overlap calculation. The distance, $D_{s}$, is the minimum distance for which the splitting separates all the branches of the bifurcation from each other (see Fig. 6b).

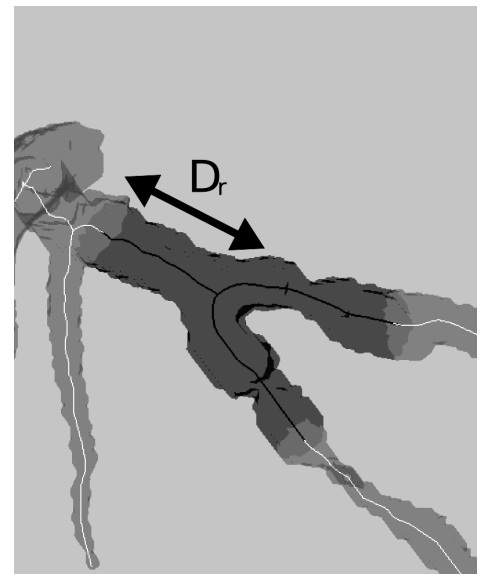

(a)

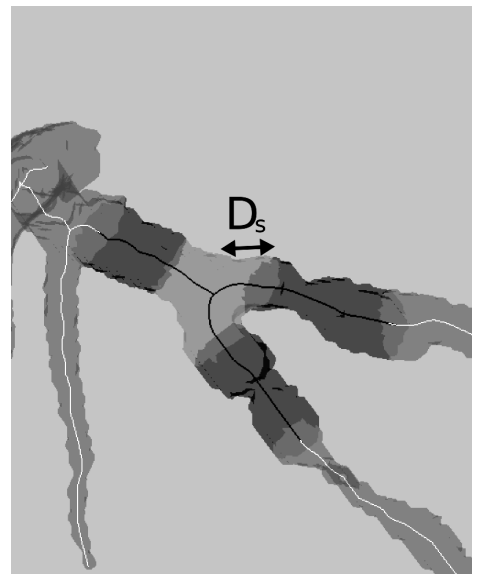

(b)

Fig. 6. Splitting of the bifurcation mesh. (a) The bifurcation and indicates the distance $D_{r}$ from the bifurcation point indicating the boundary of the region of interest. (b) The splitting of the bifurcation region at a distance $D_{s}$ to obtain the surfaces of the individual bifurcation branches.

\subsection{Foreshortening Calculation}

The amount of foreshortening of the region of interest is determined from the centerlines within this region of interest. The percentage of foreshortening $P_{f s}$ of a set of centerline pieces $C$ is calculated using the following formula taken from Chen et al. [3] .

$$
P_{f s}\left(\vec{p}, C, D_{r}\right)=\frac{\sum_{i=1}^{n} \int_{0}^{\min \left(D_{r}, T_{i}\right)}\left|\vec{c}_{i}(t) \cdot \vec{p}\right| d t}{\sum_{i=1}^{n} \int_{0}^{\min \left(D_{r}, T_{i}\right)}\left|\vec{c}_{i}(t)\right| d t} \times 100 \%
$$

Here $\vec{p}$ represent a given x-ray projection vector, $\vec{c}_{i}(t)$ is the tangent of the $i$-th centerline and $T_{i}$ is the total length of centerline $i$ in the set of $n$ centerlines in $C$. $D_{r}$ represents the length of the region of interest along each vessel. The argument $t$ represents the distance along the centerline from the bifurcation point.

\subsection{Overlap Calculation}

The projection overlap between two surface representations, $M_{l}$ and $M_{2}$, can be very quickly calculated using modern graphics hardware and the OpenGL graphics language $[10,11]$. The calculation is based on the use of stencil buffers. A stencil buffer is an off-screen image buffer that the graphics card can draw to and is used to perform per pixel comparison operations during the rendering process. The true or false return values for these comparison operations are used to modify the value of the compared pixel in the stencil buffer.

To start the overlap calculation, a virtual camera is positioned according the projection direction of the simulated x-ray system. Next, the stencil buffer is cleared and a pixel testing routine is enabled that tests if the value in the stencil buffer is equal to 0 . If the test for a certain pixel is successful the value in the stencil buffer is set from 0 to 1 . If the test fails 
nothing is changed. Next, the first surface, $M_{l}$, is rendered. Due to the enabled pixel testing, a binary silhouette of the surface projection is stored as the non-zero values in the stencil buffer (see Fig. 7b). For the next step, the pixel testing mode is changed to test for a pixel value equal to 1 . If the test is successful the stencil buffer value is increased to 2 . If it fails nothing is changed. The second surface, $M_{2}$, is rendered. The stencil buffer now contains an image of the projection of surface, $M_{l}$, as the non-zero values with the overlap of the other surface, $M_{2}$, as the pixels with value 2 (see Fig. $7 \mathrm{c}$ ). The contents of the stencil buffer can be read out and used to calculate the percentage of overlap, $P_{\text {overlap }}$.

$$
P_{\text {overlap }}=\frac{n_{2}}{n_{1}+n_{2}} \times 100 \%
$$

Where $n_{i}$ is the number of pixels with value $i$ in the stencil buffer.

The percentage of overlap can be calculated even faster. During each rendering and pixel testing step a counter, $X_{i}$, is created and increased for each successful stencil test. Requesting the counter values after each rendering step is much faster than obtaining the entire stencil buffer. This way the percentage of foreshortening can be expressed as:

$$
P_{\text {overlap }}=\frac{X_{2}}{X_{1}} \times 100 \%
$$

Where $X_{I}$ and $X_{2}$ are the number of successful stencil test for the drawing of the surface $M_{1}$ and $M_{2}$ respectively.
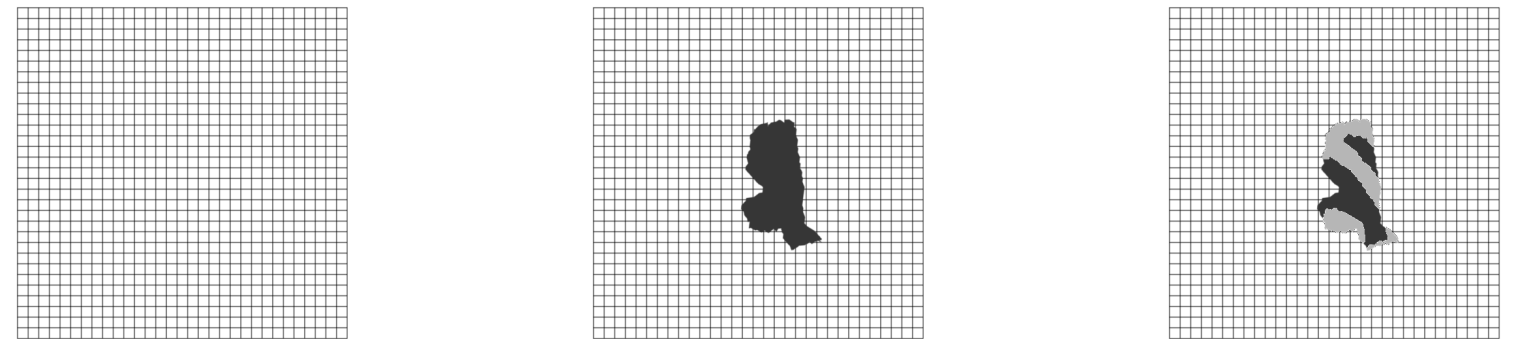

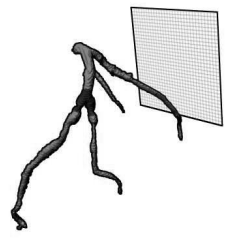

(a)

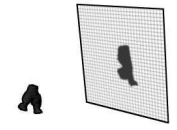

(b)

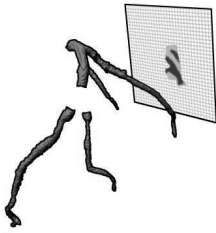

(c)

Fig. 7. Illustration of steps in the overlap calculation using stencil buffers. The top row of figures shows a representation of the state of stencil buffer for each step. The bottom row illustrates the 3D surface that is rendered. (a) The start of the calculation showing the 3D surface of the coronary tree with the selected bifurcation area and the cleared stencil buffer.

(b) The rendering of the bifurcation surface produces a binary silhouette of the bifurcation in the stencil buffer (dark).

(c) The projection of the remainder of the tree shows the overlapping areas in the stencil buffer (light).

Using this calculation method, the percentage of overlap can very quickly be calculated. For the calculation of the internal overlap, the maximum value of the three overlap calculations between the individual branches is used.

\subsection{Combining Angle Criteria}

The percentage of foreshortening, overlap and internal overlap for a bifurcation are calculated for a range of rotation and angulation angles of the x-ray system. In this study, we limited the rotations between RAO 40 and LAO 60. The minimum and maximum angulations were set to Caudal 35 and Cranial 30, respectively. These values are taken from the ranges used in clinical practice. The results from these calculations can be presented as a 2D map (see Fig. 8a-c). 
In terms of these maps, the optimal viewing angle is determined by combining the values from maps for the foreshortening, overlap and internal overlap in a new map. For this combined map, the overlap and internal overlap map are used to mask regions in the foreshortening map. This mask is constructed by finding only the regions resulting in less than $20 \%$ overlap and less than $10 \%$ internal overlap. To prevent the algorithm from choosing angles too close to regions of high overlap an additional safety zone at $10^{\circ}$ distance from these regions is added to the mask. The optimal angle is next determined as the angle with the minimum amount of foreshortening in the combined map.

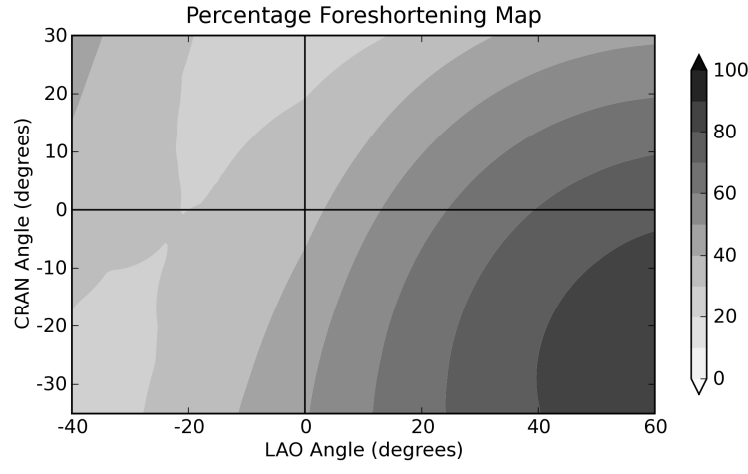

(a)

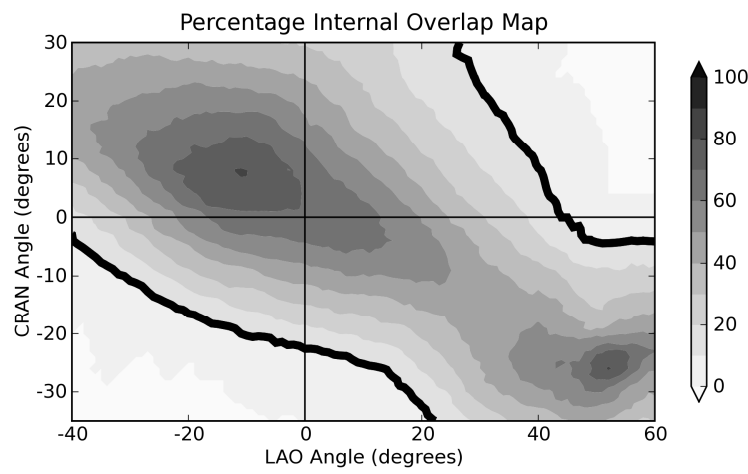

(c)

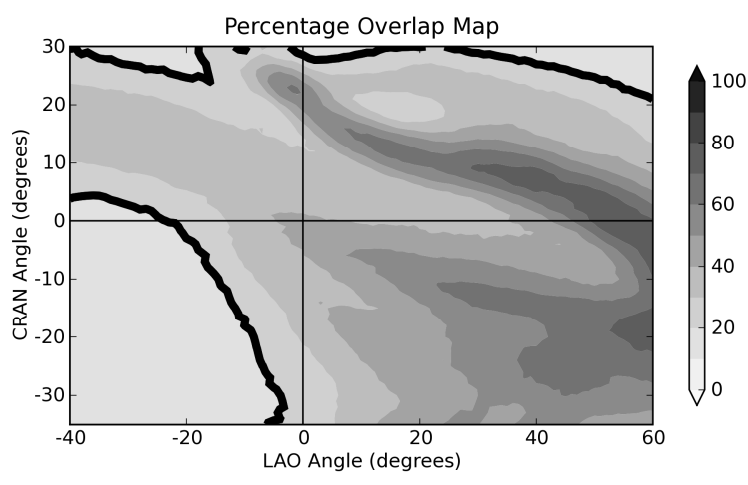

(b)

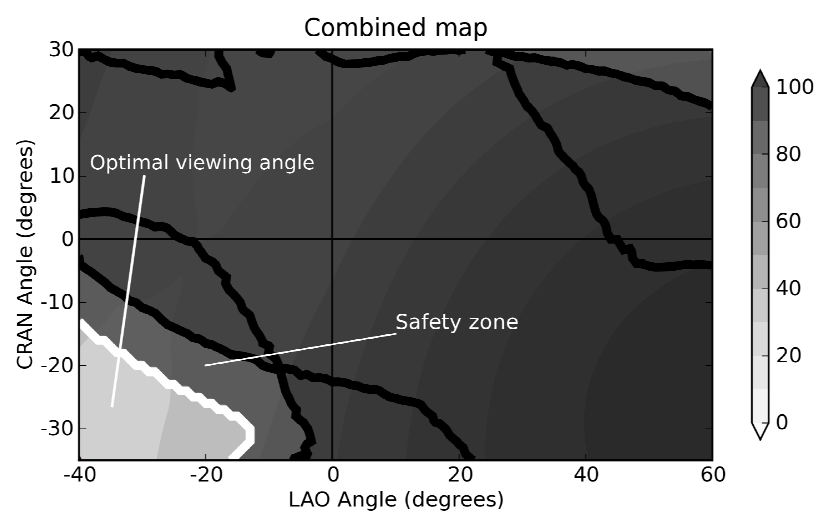

(d)

Fig. 8. Maps showing the percentage of (a) foreshortening, (b) overlap and (c) internal overlap and the (d) combined map. The dark lines indicate the boundaries of the masked regions for the different criteria. The additional safety zone and the optimal viewing angle are also indicated.

\section{VALIDATION EXPERIMENTS}

From 15 CTA datasets the left and right coronary arterial trees were segmented using the described segmentation scheme. This resulted in a total number of 83 bifurcation areas. For a number of the CT datasets, also x-ray angiographic images were available. After the segmentation of the coronary trees, a simple visual comparison was done between the real x-ray images and simulated projections from the same angle using the surface representations. For each of the 83 bifurcation points the optimal angiographic viewing angle was automatically determined.

A number of tests was performed to determine the robustness of the algorithm to some of the used parameters during the segmentation and angle determination steps. These robustness tests will be discussed later in more detail. Also, two experts (interventional cardiologists) were asked to manually set preferred viewing angles for all the bifurcation points. This was done using a computer program showing simulated x-ray projections of the extracted coronary tree from a 
viewing angle that could interactively be controlled. The obtained viewing angles of the algorithm and the experts were compared in both a quantitative and qualitative manner. The amount of resulting foreshortening for the expert set angels and automatically determined angles were calculated. Finally, one of the experts scored the projection images from angles set by the other expert and the angles determined by the algorithm. These scores indicated the clinical usefulness of the obtained projections. The scoring range was 0 to 10 with scores of 6 or above indicating clinical usable projection angles.

\subsection{Robustness tests}

Three stability tests were done to determine the sensitivity of the optimal angle algorithm to some of the parameters of the calculation. The first test was preformed to determine the sensitivity of the algorithm to the position of the coronary tree (patient) in the simulated x-ray system. Experiments were done in which the optimal angiographic viewing angle was calculated for a translated coronary tree. This was carried out for translations of $10 \mathrm{~mm}$ and $50 \mathrm{~mm}$. The translations were performed in both the positive and negative directions along the axis of the $\mathrm{x}$-ray system as depicted in figure 2. A second test was done to determine the optimal angles for a variation of sizes of the region of interest of the bifurcations. One run calculated the optimal angles for the region boundary at $D_{r}=5 \mathrm{~mm}$ distance from the bifurcation point and one for the region boundary at $D_{r}=15 \mathrm{~mm}$ distance. Finally, a test was performed to determine the influence of our used definition of the position of the bifurcation point on the calculated optimal viewing angle. This was done by calculating the centerlines of all the coronary trees using another distance criterion to position the bifurcation point along the average centerline. Two experiments were performed where criteria of $d=0.25 \mathrm{~L}$ and $d=0.75 \mathrm{~L}$ were used (see Fig. 4).

\section{RESULTS}

For each translation step of the coronary tree there were no significant differences within the results of the optimal angle deviation for the different directions. Therefore, the angle deviations for all directions were averaged. The results of the $10 \mathrm{~mm}$ and $50 \mathrm{~mm}$ coronary tree translation, as well as the results of the bifurcation size variation, and the bifurcation point repositioning are summarized in table 1.

Table 1: Optimal angle deviations (degrees) for robustness test $(\mathrm{N}=83)$.

\begin{tabular}{ll|ll|ll|ll|} 
& & \multicolumn{2}{|c|}{ Patient translation } & \multicolumn{2}{|c|}{ Bifurcation region } & \multicolumn{2}{|c|}{ Bifurcation point position } \\
& & $10 \mathrm{~mm}$ & $50 \mathrm{~mm}$ & $D_{r}=5 \mathrm{~mm}$ & $D_{r}=15 \mathrm{~mm}$ & $d=0.25 L$ & $d=0.75 \mathrm{~L}$ \\
\hline LAO & Mean & -0.1 & 0.1 & 0.1 & 1.0 & 1.2 & 0.1 \\
& Std & 1.1 & 2.4 & 25.7 & 18.3 & 17.0 & 9.8 \\
\hline CRAN & Mean & 0.1 & -0.2 & -0.7 & -0.4 & -2.2 & 0.4 \\
& Std & 1.8 & 1.7 & 16.1 & 10.1 & 13.5 & 10.4
\end{tabular}

The mean score of the algorithm during the qualitative comparison of the determined angiographic views was $8.4 \pm 1.1$. The mean score of expert A was $8.7 \pm 0.8$. In $95 \%$ of the cases the algorithm produced a clinically usable view (score $>=$ 6) as compared to $98 \%$ for expert A.

The mean percentage of foreshortening in the angiographic views of the algorithm is $25 \pm 10 \%$. For expert $\mathrm{A}$ and $\mathrm{B}$ these values are $34 \pm 15 \%$ and $35 \pm 14 \%$, respectively (see Fig. 9). There is no significant difference between the percentages of foreshortening for the two expert set angles. The algorithm set angles result in a statistically significant smaller foreshortening of 10 percentage points ( $95 \%$ confidence interval 8 to 12 percentage points) as compared to the experts. 


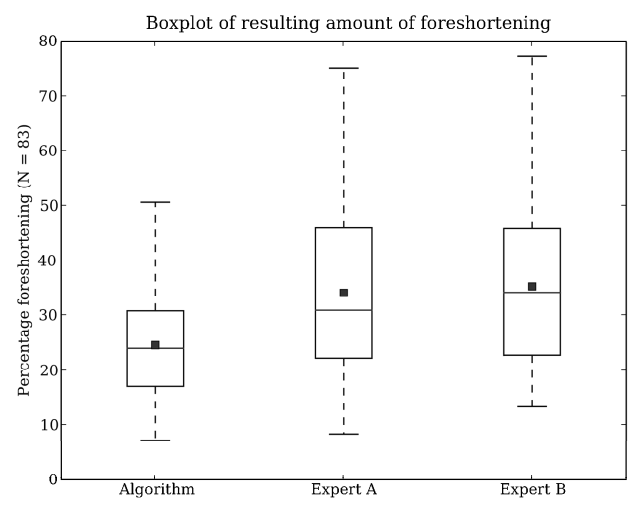

Fig. 9. Boxplot of resulting foreshortenings of chosen angles for the algorithm, expert A and expert B.

\section{CONCLUSION}

A method is presented to determine optimal viewing angles for coronary x-ray angiography based on available CTA data of a patient. The method consists of a semi-automatic segmentation step followed by the automatic determination of centerlines and bifurcation points of the coronary tree. Next, a bifurcation specific optimal viewing angle is calculated based on the minimization of foreshortening and vessel overlap. The method is able to produce clinical relevant views with less foreshortening in comparison with experts. Because of the low sensitivity to the positioning of the patient it is a potentially valuable method for the planning of angiographic interventions.

\section{REFERENCES}

[1] Dumay A.C.M., Reiber J.H.C., Gerbrands J.J., "Determination of Optimal Angiographic Viewing Angles: Basic Principles and Evaluation Study," IEEE Transactions on Medical Imaging 13(1), 13-24 (1994).

[2] Christiaens J., Van de Walle R., Gheeraert P., Lemahieu I., Taeymans Y., "Determination of optimal angiographic viewing angles for QCA," International Congress Series 1230, 909-915 (2001).

[3] Chen S.J., Carroll J.D., "3-D Reconstruction of Coronary Arterial Tree to Optimize Angiographic Visualization," IEEE Transactions on Medical Imaging 19(4), 318-336 (2000).

[4] Green N.E., Chen S.J., Hansgen A.R., Groves B.M., Carroll J.D., "Angiographic Views Used for Percutaneous Coronary Interventions: A Three-Dimensional Analysis of Physician-Determined vs. Computer-Generated Views," Catheterization and Cardiovascular Interventions 64, 451-459 (2005).

[5] Cline H.E., Thedens D.R., Irarrazaval P., Meyer C.H., Hu B.S., Nishimura D.G., Ludke S., "3D MR Coronary Artery Segmentation," Magnetic Resonance in Medicine 40, 697-702 (1998).

[6] Wood S.A., Zerhouni E.A., Hoford J.D., "Measurement of three-dimensional lung tree structures by using computed tomography," J Appl Physiol 79, 1687-1697 (1995).

[7] Marquering H.A., Dijkstra J., de Koning P.J.H., Stoel B., Reiber J.H.C, "Towards quantitative analysis of coronary CTA," The International Journal of Cardiovascular Imaging 21, 73-84 (2005).

[8] Janssen J.P., Koning G., de Koning P.J.H., Tuinenburg J.C., Reiber J.H.C., "A novel approach for the detection of pathlines in X-ray angiograms: the wavefront propagation algorithm," The International Journal of Cardiovascular Imaging 18(5), 317-324 (2002).

[9] Lorensen W.E., Cline H.E., "Marching Cubes: A High Resolution 3D Surface Construction Algorithm," Computer Graphics Proc. SIGGRAPH 21(4), 163-169 (1987).

[10] Wink O., Kemkers R., Chen S.J., Carroll J.D., "Coronary Intervention Planning Using Hybrid 3D Reconstruction," MICCAI-LNCS 2488, 604-611 (2002).

[11] Woo J.D., Neider J., David T., Schreiner D., "OpenGL Programming Guide, Version 1.2", Addison Wesley, Reading, Mass, (1999). 